Volume 1 Issue 2

December 2016

\title{
Paramedic Education - Is Dynamic Problem Based Learning The Way Forward?
}

Mark Dixon MSc

Paramedic Studies, Graduate Entry Medical School, University of Limerick, Ireland

Frank Keane BSc

Paramedic Studies, Graduate Entry Medical School, University of Limerick, Ireland

Andrew Taylor MSc

North West Ambulance Service NHS Trust, United Kingdom

\section{Recommended Citation}

Dixon M, Keane F and Taylor A. Paramedic Education - Is Dynamic Problem Based Learning The Way Forward? 2016 Dec; 1(2)

This is an Open Access article distributed under the terms of the Creative Commons Attribution-Non-Commercial-ShareAlike 4.0 International (http://creativecommons.org/licenses/by-nc-sa/4.0/), which permits use, distribution, and reproduction in any medium, provided the original work and any attributes thereof are properly cited, are distributed under the same licence, and that the work is not used for commercial purposes.

Follow the Irish Journal of Paramedicine online at www.irishparamedicine.com, on Twitter (ㅆirishjparamed) and on $\underline{\text { Facebook. }}$ 
EDUCATION

\title{
Paramedic Education - Is Dynamic Problem Based Learning The Way Forward?
}

\author{
Mark Dixon MSc ${ }^{1}$, Frank Keane BSc ${ }^{1}$, Andrew Taylor MSc ${ }^{2}$ \\ 1. Paramedic Studies, Graduate Entry Medical School, University of Limerick. \\ 2. North West Ambulance Service NHS Trust, United Kingdom.
}

Correspondence: Mark Dixon, Paramedic Studies, Graduate Entry Medical School, University of Limerick. Email: $\underline{\text { mark.dixon@ul.ie }}$

Modern Emergency Medical services and the role of the paramedic is a new departure when compared with other healthcare professions. The evolution of such emergency care services begin as recently as the 1960's with the development of responding systems and practitioners who performed patient intervention rather than the traditional transport only functions.

While military battlefield evacuation protocols stemming from as far back as the Napoleonic wars defined medical models, the patient demographic of fit young men could not accurately be transposed into a public health model. It may be argued that a United States initiative to introduce a cardiovascular survival strategy in 1964 resulted in the establishment of five Regional Medical Programs (RMP).

Although such programmes sought to challenge mortality and morbidity rates associated with heart disease, cancer and stroke RMPs promoted expansion of health care providers by expanding training for groups such as nurse practitioners, physician assistants, emergency medical technicians and effectively laying principles for the modern EMS services and education in the western world. A key component of the system promoted a philosophy of technologically advanced and intensive health care centered on academic medical and education centers.(1)

In Ireland recognition of further education development requirements resulted from the report of the 'Ambulance Service Review Group 1993'.(2) This report identified the need for service level improvements in paramedic knowledge as well as the establishment of a national regulator to oversee education standards.

While the development of clinical skills is well documented over this 50 year time frame.(3) It can be argued that paramedic education modalities have not kept pace with clinical advancements. Traditional ambulance service education followed traditional pedagogy i.e. the 'teacher' was the gatekeeper of the information and the paramedic was given this information for rote learning and subsequent regurgitation come examination. The presentation media may have changed from blackboard, through flip chart and overhead projector but essentially even the modern use of PowerPoint and Keynote etc. is a reflection of this outdated methodology. Margollis et al in 2009 stated:

"Just giving instructors a PowerPoint file fosters lack of preparation"(4).

Citing that this method of education does not keep pace as ideas are developed and practice changes with best evidence, it is too easy for the instructor to ignore the latest theory for a pre-ordained lecture structure.

With existing paramedic training based on this lecture driven paradigm albeit with the inclusion of scenario based exercises to establish practical application of theoretical models it became evident that new modalities would be required. Established training was based around systems devised by the Institute of Healthcare and Development (IHCD) and subsequently the Joint Royal College of Ambulance Liaison Committee (JRCALC). A framework for the training can be found in the British Paramedic Association document created in 2006.(5) In Ireland this process was mirrored under the auspices of the statutory regulator the Pre Hospital Emergency Care Council (PHECC) with core principles and standards reflecting the IHCD model can be found under the PHECC Education and Training Standards.(6)

The challenge is therefore how do EMS educators upgrade the educational pedagogy to match the advances in clinical and health professional skills? In 2016 the Graduate Entry Medical School of the University of Limerick launched a four year Honours Bachelor's degree in Paramedic Studies (LM103) and have adopted a modified Problem Based Learning (PBL) model to make up the shortfall in paramedic education currency.

Problem based learning has been used in medical education for a long time.(7) Doctors and nurses are experienced in this methodology of education, however, paramedic education at this level is in its infancy and PBL is something not commonly used.

Traditionally within PBL, the students are given a problem at the beginning of a week and told to present the solution at the end of the week, thus enabling self-directed learning and group interaction.(8)

Vernon et al as far back as 1993 showed that in a meta analysis across 22 systems (medicine and nursing) not only did PBL produce better graduates academically but also showed higher scoring in faculty attitudes, student mood, class attendance, academic process variables, and measures of humanism.(9) They conclude that the results generally support the superiority of the PBL approach over more traditional methods.

Therefore, a dynamic PBL modality was adopted with three escalating scenario models of delivery being the new variant. Whereas the method of using scenarios is not new (10), the idea of using the students to complete the scenario themselves appears to be a new direction for the PBL. The benefits are an enhanced team ethos and learning over and 
above those found by Willis et al.

\section{Phase 1}

Week 1-4 - During this initial introduction students are offered paper driven PBL case reviews. As group they then review the data given and begin to self illicit information, definitions, data and a add process to the case. They also develop a set of learning objectives for the week and this is supplemented by the facilitator to ensure mandatory components of the syllabus are not omitted. All sources of information are permitted including library resources and online information, with group discussion heavily promoted. The weeks conclusion is the cross presentation of the case to a peer student group with practical re-enactment of the scenario utilised as the delivery method of choice; this may then be supplemented by any other resources, models, videos etc. but it is essential this remains at the students discretion.

\section{Phase 2}

Week 5-8 - Once familiar with the general principles (including establishment of student driven ground rules) each week begins with students' being instructed to undertake a facilitated medical scenario simulation. Performance is video recorded and played back to the group for feedback. Shortfalls and good performance criteria are then identified and the students develop the weekly learning plan to address patient care improvements. Pathophysiology, anatomy, pharmacology etc. also form part of the learning and access to all resources is granted. Similar to Phase 1 the week ending is marked by practical re-enactment with debriefing offered by peer students on a parallel tract.

\section{Phase 3}

Week 8-10 - As experienced PBL operatives students are now required to again cover a range of practical scenarios and skills. Access to electronic and library resources is now removed with the knowledge held within the group deemed sufficient to work out bodily function, process, consequence etc. End of week presentations to the parallel peer group rely completely on student interaction and delivery with others. No electronic or written resources are permitted representing a true reflection of the acquired knowledge.

\section{Discussion}

As the programme is still within it's first cycle comparative studies versus conventional methodology are not yet possible. However student reaction to this initiative has been positive and all learning objectives have been met thus far. In the opinion of the faculty, we would strongly endorse that PBL is more effective than learning based on established disciplines in the traditional curriculum.(11-13) Moreover, PBL conforms to the principles of adult learning and cognitive science.

As identified by Charlin et al. (12) there are four principles identified which enhance adult learning and improve the efficacy of learning:

1. learners are active processors of information;

2. prior knowledge is achieved and new knowledge is built upon it;

3. knowledge is acquired in a meaningful context;

4. learners have opportunities for elaboration and organization of knowledge.

It is the opinion of the authors that a dynamic PBL model for paramedic education is not only appropriate in terms of acquisition of knowledge but that students have ownership of this learning and the education takes place within a paramedic context.

Further study will be required at the end of the programme to ascertain the effectiveness of the PBL approach to paramedic education in general and the new style of PBL used by Paramedic Studies within the Graduate Entry Medical School, University of Limerick. That said, anecdotal evidence thus far would suggest that, thankfully, the end of 'Death by Powerpoint' is close at hand.

\section{References}

1. Shah M (2006) The Formation of the Emergency Medical Services System. American Journal of Public Health;96 (3):414-423. doi:10.2105/AJPH.2004.048793.

2. Department of Health (1993) Report of the Review Group on the Ambulance Service. Available from http:// www.lenus.ie/hse/handle/10147/81141

3. Radcliffe J, Heath G (2006) Implications of the changing role of the Ambulance Paramedic for the interpretation of inappropriate calls: a review of the literature. Available from http://www4.ntu.ac.uk/nbs/document_uploads/99206.pdf

(Accessed Nov 25, 2016).

4. Margolis GS Romero G, Fernadaz M, Studnek J (2009) Education and Practice. Prehospital Emergency Care Oct 1, 2009 .

5. British Paramedic Association (2006) A curriculum framework for ambulance education.

6. Pre Hospital Emergency Council (2014) Education and Training Standards. Naas, Ireland.

7. Barrows H, Tamblyn M (1980) Problem-based learning Springer Publishing Company.

8. Willis S, Jones A, McArdle P, O’Neill PA (2003) A qualitative study of the attitudes towards teamwork of graduates from a traditional and an integrated undergraduate medical course. Advances in Health Sciences Education 8 (2) 139-148.

9. Vernon D, Blake R (1993); Does problem-based learning work? A meta-analysis of evaluative research. Academic Medicine. 68(7):550-63.

10. Barrett T, Moore S (Eds) (2011) New approaches to Problem Based Learning. Routledge New York.

11. Pallie W, Carr D (1987). 'The McMaster Medical Education Philosophy in Theory, Practice an Historical Perspective'. Medical Teacher, 9, 59-71. 
12. Charlin B, Mann K, Hansen P (1998) The many faces of problem-based learning: a framework for understanding and comparison Medical Teacher, 20 (4), pp. 323-330.

13. Farrow R, Norman G (2003) The effectiveness of PBL: the debate continues. Is meta-analysis helpful?, Medical Education, 10.1046/j.1365-2923.2003.01725.

Author contributions: MD, FK and AT were principal authors of the manuscript, and all contributed to the final editing.

Source of support/funding: None.

Conflict of interest: MD is a member of the IJP Editorial Board.

Provenance and review: Not commissioned, not peerreviewed.

This is an Open Access article distributed under the terms of the Creative Commons Attribution-Non-Commercial-ShareAlike 4.0 International (http:// creativecommons.org/licenses/by-nc-sa/4.0/), which permits use, distribution, and reproduction in any medium, provided the original work and any attributes thereof are properly cited, are distributed under the same licence, and that the work is not used for commercial purposes. Content copyright remains with the authors, who grant the IJP a licence to reuse and distribute. 